\title{
Role of Susceptibility Weighted Imaging in Cerebellopontine Angle Schwannoma Vs Meningioma
}

\author{
Saravanan K ${ }^{1}$, E.A. Parthasarathy ${ }^{2}$, Abubacker Sulaiman Farook ${ }^{3}$, Praveen Sridharan ${ }^{4}$, Gopalakrishnan $^{5}$, \\ Rajamani Anand ${ }^{6}$ \\ ${ }^{1}$ Final Year Post Graduate Student, ${ }^{2}$ Assistant Professor, ${ }^{3}$ Professor, ${ }^{4}$ Assistant Professor, ${ }^{5}$ Assistant Professor, ${ }^{6}$ Assistant \\ Professor, Department of Radiology and Imaging Sciences, Chettinad Hospital and Research Institute, India
}

Corresponding author: Dr. E.A. Parthasarathy, Assistant Professor, Department of Radiology and Imaging Sciences, Chettinad Hospital and Research Institute, Kelambakkam, Kanchipuram- 603103, Tamil Nadu, India.

DOI: 10.21276/ijcmsr.2018.3.2.6

How to cite this article: Saravanan K, E.A. Parthasarathy, Abubacker Sulaiman Farook, Praveen Sridharan, Gopalakrishnan, Rajamani Anand. Role of Susceptibility Weighted Imaging in Cerebellopontine Angle Schwannoma Vs Meningioma. International Journal of Contemporary Medicine Surgery and Radiology. 2018;3(2):B20-B23.

\section{A B S T R A C T}

Introduction: Vestibular schwannomas may sometimes be difficult to differentiate from cerebellopontine angle (CPA) meningiomas. Identifying foci of blooming on Susceptibility Weighted Imaging (SWI) has the potential to differentiate Vestibular schwannomas from CPA meningiomas. Hence; we planned the present study to assess the role of susceptibility weighted imaging in cerebellopontine angle schwannomas vs meningiomas cases.

Material and methods: In this prospective study of 32 consecutive patients of CPA tumors (schwannomas and meningiomas), SWI sequence is done along with all other routine basic sequences in conventional MRI. For all cases histopathological diagnosis was compared after surgery.

Result: In our prospective study 32 patients were included, out of which 23 patients are diagnosed as Vestibular schwannoma and 9 patients as meningiomas. Microhemorrhage was noted in T2* sequence (SWI) as blooming foci in 22 cases out of 32 which gives a potential diagnosis of vestibular schwannomas and rest of 9 cases shows no blooming and they are diagnosed as meningiomas. All patients underwent surgery and Histopathological diagnosis was compared with our study. Statistical analysis shows sensitivity of $95.6 \%$, specificity of $100 \%$, positive predictive value of $100 \%$ and negative predictive value of $90 \%$ and diagnosing accuracy of $96.8 \%$ for T2* weighted SWI sequence for microhemorrhages in vestibular schwannomas. Conclusion: MR imaging demonstrates microhemorrhages in most of VS in T2* -susceptibility weighted imaging. This helps to differentiate Vestibular schwannomas from CPA meningiomas. T2* - susceptibility weighted imaging should be used as a routine sequence to evaluate CPA tumors.

Key words: Vestibular Schwannomas, Susceptibility Weighted Imaging, Cerebellopontine Angle

\section{INTRODUCTION}

Cerebellopontine angle (CPA) tumors accounts for $5-10 \%$ of all intracranial tumors in adults and $1 \%$ of all paediatric intracranial tumors. In which 80 to $90 \%$ constitute Vestibular schwannomas (VS) and 10 to $15 \%$ meningiomas. ${ }^{1}$ Vestibular schwannomas may sometimes be difficult to be differentiated from cerebellopontine angle meningiomas. Magnetic resonance imaging is a vital tool to assess cerebellopontine angle tumors and to distinguish vestibular schwannomas from meningiomas. Demonstration of intratumoral microhemorrhages with T2* - susceptibility weighted imaging shows characteristic feature of identifying vestibular schwannomas from that of meningiomas. ${ }^{2}$ In most cases, MR imaging and computed tomography (CT) display characteristic festures of meningioma's. Acoustic neuromas are routinely round masses in the cerebellopontine cistern that emerge from the internal auditory canal, widen the porus, and grow posteriorly be- cause of the anterior limit represented by the cisternal segment of the facial nerve. They can be heterogeneous due to cystic components. Conversely, meningiomas are usually hemispheric, semilunar masses with a broad petrous base to which they are attached and are usually asymmet- ric to the internal auditory canal. ${ }^{3}$ The aim of present study was to assess the role of susceptibility weighted imaging in cerebellopontine angle schwannomas vs meningiomas cases.

\section{MATERIAL AND METHODS}

In our prospective study from Jun 2015 to Dec 2017, conducted in the Department of Radiology in Chettinad hospital, we included 32 patients. After obtaining oral and written informed consent and ethical clearance, the patients were subjected to MR imaging with 1.5 Tesla SIGNA GE HDxt MRI scanner with an 8 channel NV (NAVIGATOR) radiofrequency coil. The bore size of the machine is $60 \mathrm{~cm}$. Image acquisition parameter are shown in Table 1. 
Histopathologic confirmation of the diagnosis was done in all cases.

Approximately 2 and half minutes was the acquisition time. We performed the post processing, followed by generation of thick minimum intensity projection (mIP) slabs. We, then, reviewed the images obtained on a DS3000 Impax workstation. For image analysis purpose, we used an image matrix of 374512 and FOV of $220 \mathrm{~mm} 240 \mathrm{~mm}$. The presence of intratumoral microhemorrhages was identified by hypointense dots on T2* MRI similar to that of cerebral microhemorrhages. ${ }^{2-4}$

\section{STATISTICAL ANALYSIS}

All the results were analyzed by SPSS software version 17.0. Multivariate regression curve were used for assessment of level of significance.

\section{RESULTS}

In our prospective study 32 cases were included, out of which 23 patients were diagnosed as Vestibular schwannoma and 9 patients were diagnosed as meningiomas (fig.1). Out of 23 patients of vestibular schwannoma, 8 patients were women and 15 were men. In 9 cases of meningiomas 6 patient were women and 3 patients were men (fig.2). The age range of vestibular schwannomas are 21 to 55 years were as for meningiomas 40 to 60 years. According to the location of the CPA tumors in vestibular schwannomas 17 cases presented on right and 6 cases on left side whereas in meningiomas 6 cases on right side and 3 on left side (fig.3). Distribution of

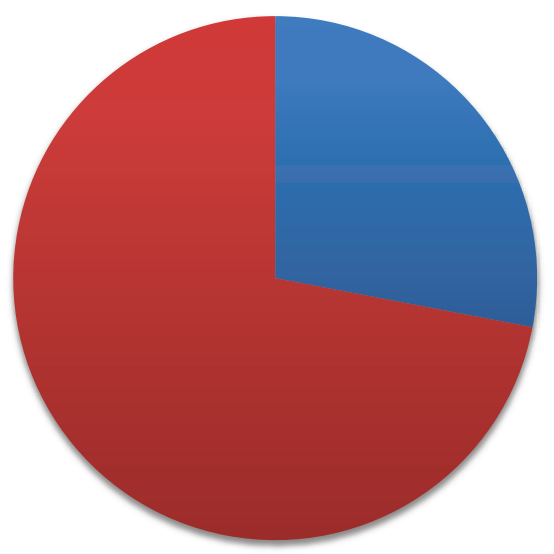

Maningiomas Vestibular Schannomas

Figure-1: PIE diagram showing distribution of sample type of CPA tumor lesions, 22 cases were solid and 1 case is cystic in vestibular schwannomas whereas in meningiomas all the 9 cases were solid. Among 23 cases of vestibular schwannomas, IAC extension is noted in all cases. In T1 weighted imaging sequence tumor shows hypointense in 22 cases and isointense in 1 case. The matrix of tumor shows heterogeneously hyperintense on T2 weighted imaging in all the vestibular schwannoma cases. Intense enhancement is noted in 17 cases and moderate enhancement in 6 cases. Microhemorrhage were noted in $\mathrm{T}^{*}$ sequence (SWI) as blooming in 22 cases out of 32 which gives a potential

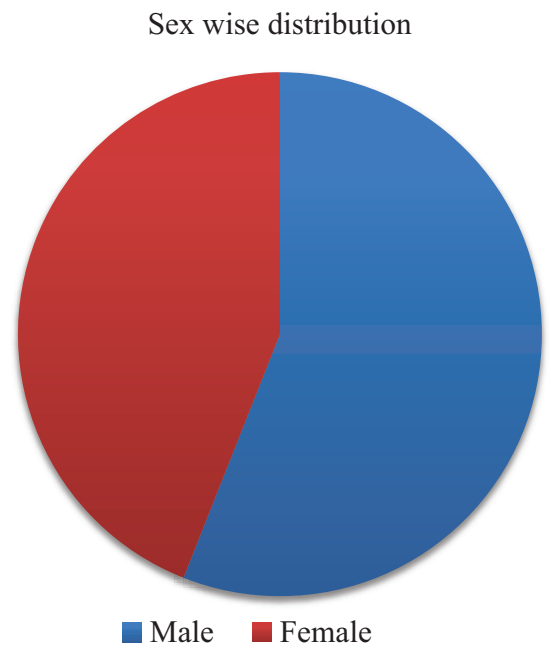

Figure-2: PIE diagram showing sex distribution of patients

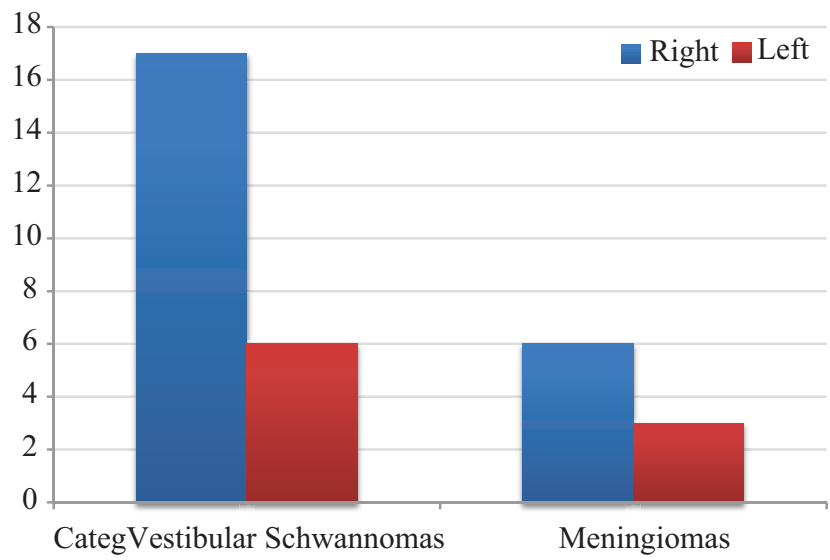

Figure-3: bar diagram showing distribution of sample based on location

\begin{tabular}{|c|c|c|c|c|c|c|}
\hline Parameter & T2 Flair & T2 TSE & T1 SE & T1 SE & T1 SE & SWI \\
\hline Mode of acquisition & $2 \mathrm{D}$ & $2 \mathrm{D}$ & $2 \mathrm{D}$ & $2 \mathrm{D}$ & $2 \mathrm{D}$ & $3 \mathrm{D}$ \\
\hline Plane & Axial & Axial & Axial & Sagittal & Axial & Axial \\
\hline FOV $(\mathrm{mm})$ & 24 & 24 & 24 & 26 & 24 & 24 \\
\hline TR & 6500 & 4000 & 800 & 825 & 830 & 62 \\
\hline TE & 800 & 650 & 75 & 80 & 90 & 41 \\
\hline Matrix & $320 * 224$ & $320 * 256$ & $320 * 192$ & $320 * 192$ & $320 * 224$ & $352 * 160$ \\
\hline NEX & 2 & 2 & 2 & 2 & 1 & 1 \\
\hline FAT-SAT & No & No & No & No & Yes & No \\
\hline Slice thickness $(\mathrm{mm})$ & 4 & 4 & 4 & 4 & 4 & 3 \\
\hline Timing (min) & 3.28 & 1.45 & 1.05 & 1.15 & 3.45 & 3.00 \\
\hline
\end{tabular}




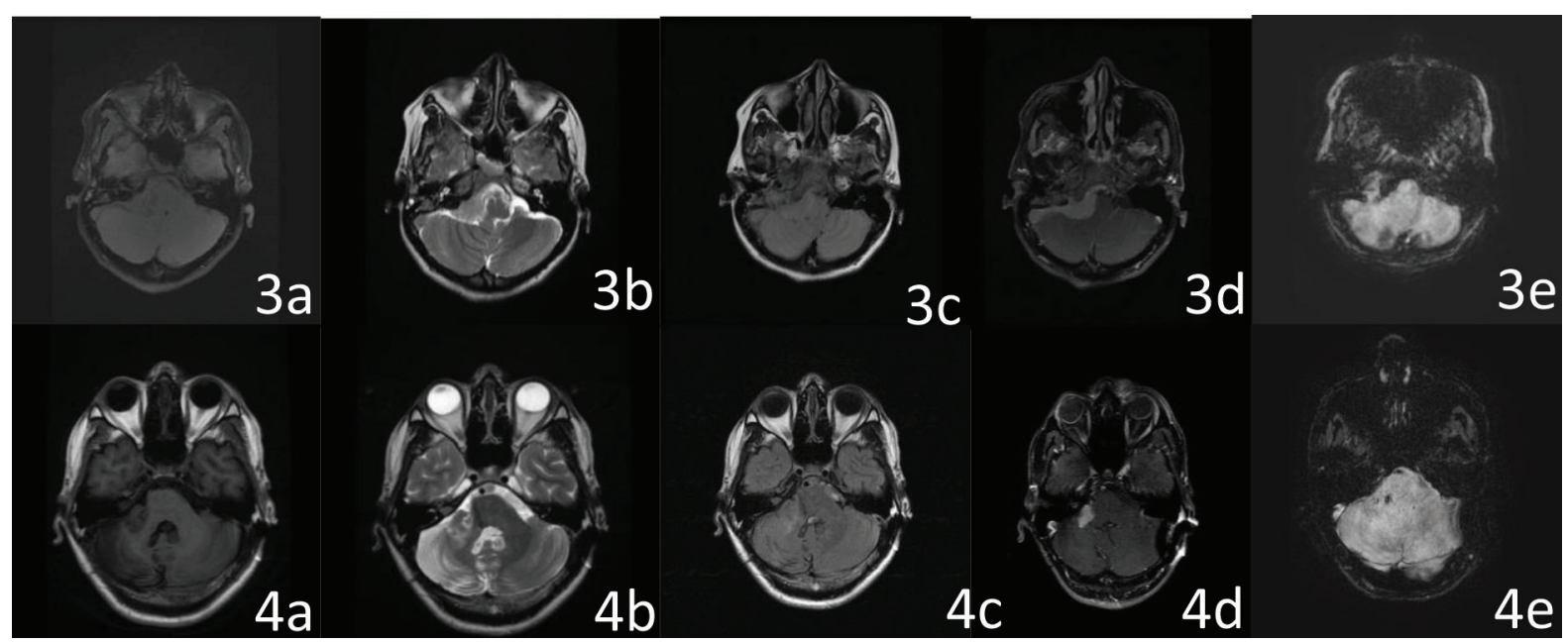

Figure-3a: T1WI shows lesion isointense to grey matter in right CPA Fig.3b: T2WI shows lesion which is iso-hyperintense to grey matter in right CPA Fig.3c: T2 FLAIR shows iso-hypointense lesion in right CPA Fig.3d: T1 C+: shows intense and homogeneous post contrast enhancement of the lesion Fig.3e: SWI shows no blooming within the lesion Figure 4a: Images of Vestibular Schwannomas: T1WI shows iso-hypointense lesion in right CPA Figure 4b: Images of Vestibular Schwannomas: T2WI shows heterogeneously hyperintense lesion in right CPA Figure 4c: Images of Vestibular Schwannomas: T2 FLAIR shows isointense to grey matter lesion in right CPA Figure 4d: Images of Vestibular Schwannomas: T1 C+: intense enhancement of the lesion in right CPA Figure 4e: Images of Vestibular Schwannomas: SWI shows foci of blooming (microhemorrhages) within the lesion

diagnosis of vestibular schwannomas and rest of 9 cases shows no blooming and they are diagnosed as meningiomas. CT has been done to exclude calcification in all cases.

Among 9 cases of meningiomas, IAC extension is noted in 2 cases. The dural tail was seen in 6 cases and flat base against dura was noted in 3 cases. None of the case showed microhemorrhages on T2* weighted SWI sequence. Uniform enhancement was noted in all case. No obvious calcification noted in the CT.

Statistical analysis shows sensitivity of $95.6 \%$, specificity of $100 \%$, positive predictive value of $100 \%$ and negative predictive value of $90 \%$ and diagnosing accurarcy of $96.8 \%$ for T2* weighted SWI sequence for identifying microhemorrhages in vestibular schwannomas.

\section{DISCUSSION}

The term Cerebellopontine angle (CPA) was first described by Henneberg and Koch in 1902, they reported two individuals with bilateral acoustic neuromas occurring in the location they described (in German) as kleinhirnbruchen-winkel (kleinhirn = cerebellum; bruchen $=$ pons or bridge; and winkel $=$ angle). ${ }^{1}$ Cerebellopontine angle (CPA) is lined or covered by the meningeal layers and added to that of CSF, nerves, vessels, and possibly embryologic remnants. CPA lesions can be arising from any of these structures. CPA lesions are categorized into three major groups 1) Lesions originating in CPA, 2) lesion primarily located in close anatomical sites and extending to the CPA and 3) Intraventricular and brain stem pathologies showing exophytic expansion to the CPA. About 6 to $10 \%$ of all intracranial tumors arise in or involve the cerebellopontine angle. ${ }^{5}$ The first most common CPA tumors are vestibular schwannomas, which constitute 80 to 90\%. The next most common CPA tumor constitute 10 to $15 \%$ is meningiomas. Clinically audiovestibular disturbance (hearing loss, dysequilibrum, and tinnitus) are more common symptom of vestibular schwannomas as compared with meningiomas. Hearing loss is found in 50 to $80 \%$ of patient with meningiomas as compared with almost all patient with vestibular schwannomas.

The globular shape of the tumor with dilatation of the internal auditory canal in association of canalicular component and formation of acute angle by anterolateral or posterolateral tumor border with the adjacent petrous bone is highly suggestive of vestibular schwannomas. Most meningiomas are large, sessile and oval in shape with a broad dural attachment to the petrous bone forms an obtuse angle. Hyperostosis and calcifications are more frequently seen in meningiomas and involvement of the internal auditory canal (IAC) is less frequent in meningiomas than vestibular schwannomas. ${ }^{4,5}$

Vesibular schwannomas are isointense to hypointense to brain in T1W images and hyperintense on T2W images. They may be hetrogenous due to cystic degeneration and haemorrhage. They are usually round or oval in shape and taper along the axis of the internal acoustic meatus like 'ice cream cone appearance'. Meningiomas are usually isointense to brain in both $\mathrm{T} 1$ and T2 weighted images and with contrast, intense and homogeneous enhancement is seen. Even dural tail of enhancement may also be present. Mostly they do not show extension into the internal acoustic meatus and usually have a broad base against petrous bone. ${ }^{6,7}$

In our study all patients underwent MRI brain are subjected to the $\mathrm{T}^{*}$ sequence (SWI) in which blooming are noted in 22 cases out of 32 cases which gives a potential diagnosis of vestibular schwannomas due to presence of microhemoarrhages and rest of 9 cases shows no blooming and they are considered to be meningiomas. Hence Intratumoral microhemorrhages noted on T2* weighted SWI sequence holds a potential diagnosis for vestibular schwannomas and helps to differentiate from that of the meningiomas 
in cerebellopontine tumors. $\mathrm{CT}$ is done routinely to all preoperative patient to exclude calcification in all vestibular schwannomas cases. The treatment and operative approach varies for both vestibular schwannomas and meningiomas, so accurate preoperative diagnosis is evident to differentiate this twoCPA tumors because the outcome with preservation of hearing is better in meningiomas. Similar studies have been done by other authors with T2* Weighted GradientEcho Imaging to differentiate vestibular schwannomas and meningiomas, but we have used susceptibility weighted imaging to differentiate these two lesions. Meningiomas are known to be richly vascular tumors and may occasionally present with intratumoral, subarachnoid, or subdural hemorrhage, particularly with angioblastic and meningotheliomatous meningiomas. ${ }^{8} 9$ Cystic changes in meningiomas have been associated with hemorrhage and aggressive behavior.Focal concentrations of thin-walled vessels and endothelial channels have been recognized close to the gross bleeding sites in meningiomas. Arteriolar hyalinization has been also recognized in meningiomas presenting with hemorrhage. ${ }^{10-12}$ Mishra A et al in 73 patients, conducted MRI with susceptibility weighted imaging sequence along with other routine used techniques. All the subjects were analyzed for the occurrence of foci of blooming inside the tumor on susceptibility weighted imaging. In all the cases, diagnosis was confirmed by assessment of histopathology reports. In the vestibular schwannoma group, there were overall of 59 patients, while in the meningioma group, there were overall of 14 patients. On assessing the Susceptibility weighted imaging results; we observed the presence of blooming (due to microhemorrhages) in 100 percent cases of cerebellopontine angle schwannomas. 13 cases showed no blooming within the lesion in the meningioma group, while single case demonstrated blooming which was established to be due to calcification on CT study. Under the light of above results, the authors concluded that in making accurate pre-operative differentiation of vestibular schwannoma from meningioma, susceptibility weighted imaging is a significant useful tool. ${ }^{13}$

\section{CONCLUSION}

Conventional MR imaging demonstrates microhemorrhages in most of VS in T2* -susceptibility weighted imaging. This helps to differentiate Vestibular schwannomas from CPA meningiomas. T2* - susceptibility weighted imaging should be used as a routine sequence to evaluate CPA tumors.

\section{REFERENCES}

1. Serebellopontin Bölgenin Nadir Görülen Lezyonlar, Rare Lesions of the Cerebellopontine Angle, Turkish Neurosurgery 2010;20(1):390-397.

2. K. Thamburaj V.V. Radhakrishnan, Intratumoral microhemorrhages on T2* Weighted Gradient-Echo Imaging Helps Differentiate Vestibular Schwannoma From Meningioma, AJNR Am J Neuroradiol Mar 2008;29(3):552-57.

3. Bonneville F, Sarrazin JL, Marsot-Dupuch K, Iffenecker C, Cordoliani YS, Doyon D: Unusual lesions of the cerebellopontine angle: Asegmental approach.
Radiographics 2001;21(4): 419-438.

4. Jacob Bertram Springborg Lars Poulsgaard, Nonvestibular Schwannoma Tumors in the Cerebellopontine Angle: A Structured Approach and Management Guidelines, January 3, 2008.

5. Kunwarpal Singh, Mohit Preet Singh, Role of Magnetic Resonance Imaging in Evaluation of Cerebellopontine Angle Schwannomas, Indian J Otolaryngol Head Neck Surg 2015; 67(1):21-27.

6. Zamani AA: Cerebellopontine angle tumors: Role of Magnetic resonance Imaging. Top Magn Reson Imaging 2000;11(2):98-107.

7. Glasscock ME, Minor LB, McMenomey SO. Meningiomas of the cerebellopontine angle. In: Jackler R, Brackmann D, eds. Neurotology. St. Louis: Mosby 1994:795-823.

8. Tali ET, Yuh WT, Nguyen HD, et al. Cystic acoustic schwannomas: MR characteristics. AJNR. 1993;14(5):1241-1247.

9. Valvassori GE, Garcia Morales F, Palacios E, Dobben GE. MR of the normal and abnormal internal auditory canal. AJNR. 1988;9(1):115-119.

10. Valavanis A, Schubiger $O$, Naidich T. Clinical imaging of the cerebellopontine angle. Berlin: Springer-Verlag; 1987.

11. Bartels LJ, Arrington JR. Rare tumors of the cerebellopontine angle. In: Jackler RK, Brackmann D, editors. Neurotology. St. Louis: Mosby; 1994. pp. 835861.

12. Press GA,HesselinkJR.MRimaging of cerebellopontine angle and internal auditory canal lesions at $1.5 \mathrm{~T}$. AJR. 1988;150(6):1371-1381.

13. Mishra A1, Thomas B2, Kapilamoorthy TR2. Susceptibility weighted imaging - a problem-solving tool in differentiation of cerebellopontine angle schwannomas and meningiomas. Neuroradiol J. 2017;30(3):253-258.

Source of Support: Nil; Conflict of Interest: None

Submitted: 25-04-2018; Published online: 30-04-2018 